Palavras chave:

Fragmentos

Métricas

Área de preservação permanente

Corredor ecológico

Efeito de borda

Histórico:

Recebido 21/05/2012

Aceito 02/02/2015

Keywords:

Fragments

Metrics

Permanent Protected Areas

Ecological Corridors

Edge effect

Correspondência: rossiallan@gmail.com

DOI:
Rossi Allan Silva', José Aldo Alves Pereira', Dalmo Arantes de Barros', Luís Antônio Coimbra Borges', Marcelo Dias Teixeira', Fausto Weimar Acerbi-Jr.'

\section{AVALIAÇÃO DA COBERTURA FLORESTAL NA PAISAGEM DE MATA ATLÂNTICA NO ANO DE 20I0, NA REGIÃO DE OURO PRETO - MG}

RESUMO: Objetivou-se, neste trabalho, avaliar o estado de conservação atual da paisagem na região de Ouro Preto, Ouro Branco e Mariana, MG, correspondendo à áreas situadas no alto das bacias dos rios das Velhas, do Carmo, Gualaxo do Sul e ribeirão da Colônia. A região possui diferentes Unidades de Conservação (UCs), localizadas no domínio fitogeográfico da Mata Atlântica. Utilizaram-se imagens RapidEye AG (jun/20I0) com $5 \mathrm{~m}$ de resolução espacial. A classificação do uso do solo para obtenção das métricas da paisagem foi dividida em três classes: Floresta Estacional Semidecidual Montana, Vegetação natural 2 e Uso Antrópico. Os resultados apontaram que a área de estudo encontra-se bem preservada, principalmente dentro das UCs, onde se localizam os grandes fragmentos, principais para a conservação; as formações naturais presentes na área de estudos corresponderam a 87,08\%; as métricas mostraram que a distância média entre os fragmentos (262) é 72, $18 \mathrm{~m}$; a forma e o efeito de bordas nos fragmentos apresentaram-se adversas à preservação da biodiversidade; fortes pressões antrópicas: área urbana, solo exposto, minerações etc., estão presentes em maior intensidade fora da Zona de Amortecimento (ZA) do Parque Estadual do Itacolomi; pontos vulneráveis à conservação encontram-se, em grande parte, próximos às áreas de pastoreio e de mineração, com destaque para as áreas de campo das UCs de Ouro Branco e de pastoreio dentro da APA Estadual Cachoeira das Andorinhas.

\section{FOREST COVER EVALUATION IN THE ATLANTIC FOREST LANDSCAPE OF THE OURO PRETO REGION - MG, IN THE YEAR 2010}

ABSTRACT: This study aimed to evaluate the current state of conservation and the landscape connectivity in the area covering part of the counties of Ouro Preto, Mariana and Ouro Branco, MG. This area is located at the upper course of the Velhas river, Carmo river, Gualaxo do Sul river and Colônia creek. The region belongs to the Mata Atlantica phytogeographic ecosystem and encompasses different Conservation Units (CUs). RapidEye AG (Jun/20l0) with $5 \mathrm{~m}$ of spatial resolution images were used. The study area was divided into three classes: Seasonal semideciduous montane forest; Natural vegetation 2; Anthropic use. The results showed that the study area is well preserved, particularly inside the CUs where the fragments are bigger and are considered priority sites for conservation. The natural formations of the region corresponded to $87.08 \%$ of the study area. The metrics showed that the average distance between the 262 fragments is $72.18 \mathrm{~m}$. The shape of the fragments do not favor the preservation of biodiversity, because they are complex and irregular (fractal). The application of edge effects initially increases the fragmentation and, subsequently reduces the size of individual vegetation spots, smoothing their complex shapes. Strong anthropic pressures are present outside the buffer zone of the State Park of Itacolomi. The weak points are those surrounding pastoral and mining areas, mainly field areas of CUs in Ouro Branco and pasture areas inside the Cachoeira das Andorinhas State APA.

' Universidade Federal de Lavras - Lavras, Minas Gerais, Brasil 


\section{INTRODUÇÃO}

A ecologia da paisagem tem sido tema de relevante importância para a definição de estratégias de ocupação e uso conservacionista das bacias hidrográficas. No Brasil, o processo de fragmentação aumentou significativamente nas últimas décadas, e a consequente perda de habitats se configura como uma das maiores ameaças a conservação da biodiversidade (TABARELLI; GASCON, 2005).

As transformações causadas pelo homem, na paisagem, são expressivas e devem ser criteriosamente avaliadas, para que seja possível compatibilizar suas atividades. Para Metzger (200I), o homem está na origem dos problemas e também nas resoluções.

As Unidades de Conservação (UC) da região de Ouro Preto estão situadas em locais estratégicos para a recarga de importantes mananciais. A correta gestão dos ecossistemas locais é de extrema importância para o bem estar de milhares de pessoas, o que torna a região prioritária para a conservação. A fragmentação da paisagem torna a localização das UCs dentro das bacias hidrográficas de suma importância e para Mouton e Souza (2006) esta situação espacial determina, fortemente, como a conectividade hidrológica será afetada, assim como a transferência dessas modificações rio abaixo.

Em Minas Gerais, a Mata Atlântica possui menos de $10,04 \%$ de seu território original, porém, no caso das cidades de Ouro Preto, Ouro Branco e Mariana, possuem valores acima dessa média, respectivamente
$31,22 \%, 24,8 \%$ e $23,74 \%$ (INSTITUTO DE PESQUISAS ESPACIAIS; FUNDAÇÃO SOS MATA ATLÂNTICA INPE, 20II). Esses municípios merecem destaque, em razão da sua localização no alto de importantes afluentes das bacias de grandes rios como o São Francisco e o Doce, tornando-se responsáveis pela recarga de mananciais de importantes centros urbanos, como por exemplo, Belo Horizonte.

Dessa forma, neste estudo, objetivou-se avaliar a estrutura da paisagem nas bacias hidrográficas situadas no alto do rio das Velhas, rio do Carmo, rio Gualaxo do Sul e ribeirão da Colônia, na região de Ouro Preto - MG.

\section{MATERIAL E MÉTODO}

A área do estudo abrange parte dos municípios de Ouro Preto, Ouro Branco e Mariana - MG, a paisagem é composta por UCs, conforme a Tabela I e a Figura I.

As UCs e áreas protegidas compõem sub-bacias do domínio fitogeográfico da Mata Atlântica, as quais, segundo Carvalho (2008), utilizando a classificação descrita por Oliveira Filho et al. (2006) e Veloso et al. (199I), contêm as fisionomias de florestas estacionais semideciduais montanas, campo e campo rupestre. O clima, segundo a classificação de Köppen-Geiger é Cwa, nas áreas de menor cota e Cwb nas mais elevadas. A precipitação média anual varia entre $1.400 \mathrm{~mm}$ a $1.600 \mathrm{~mm}$ (FUNDAÇÃO JOÃO PINHEIRO, 1975). Os aspectos pedológicos da área de estudo possuem três classes principais: Cambissolos, Latossolos e Neossolo Litólicos (UFV-CETEC-UFLAFEAM, 2010).

TABELA 1 Unidades de Conservação e suas respectivas áreas no local de estudo na região de Ouro Preto, MG.

TABLE 1 Conservation Units and their respective areas at the study site in the Ouro Preto region, MG.

\begin{tabular}{|c|c|c|c|}
\hline Unidades de Conservação & Cidade & Tipo & Área da UC (ha) \\
\hline 1. Área de Proteção Ambiental (APA) Estadual Cachoeira das Andorinhas & OP & UUS & $14.269,57$ \\
\hline 2. Parque Estadual da Serra (PES) de Ouro Branco & OP/OB & UPI & $7.523,32$ \\
\hline 3. Parque Estadual do Itacolomi (PEI) & MR/OP & UPI & $5.996,48$ \\
\hline 4. Floresta Estadual do Uaimií & OP & UUS & $4.292,53$ \\
\hline 5. Monumento Natural Estadual (MNE) do Itatiaia & OP/OB & UPI & $3.216,90$ \\
\hline 6. Área de Proteção Especial Estadual (APEE) Veríssimo & OB & UUS & $1.979,56$ \\
\hline 7. Parque Natural Municipal das Andorinhas & OP & UPI & 559,59 \\
\hline 8. APA Seminário menor de Mariana & MR & UUS & 352,44 \\
\hline 9. Estação Ecológica do Tripuí (EET) & OP & UPI & 298,72 \\
\hline 10. Área de Preservação Fazenda ou Serra da Brígida & OP & Outros* & 154,39 \\
\hline 11. Reserva Particular do Patrimônio Natural (RPPN) Fazenda Capanema & SB/OP & UUS & 299,36 \\
\hline 12. Parque Arqueológico Morro da Queimada & OP & Outros* & 68,76 \\
\hline
\end{tabular}

Unidades de Uso Sustentável (UUS); Unidades de Proteção Integral (UPI); Ouro Preto (OP); Ouro Branco (OB); Mariana (MR); Santa Bárbara (SB).

*Áreas não regulamentadas de relevante interesse ecológico e, portanto, sem enquadramento. 


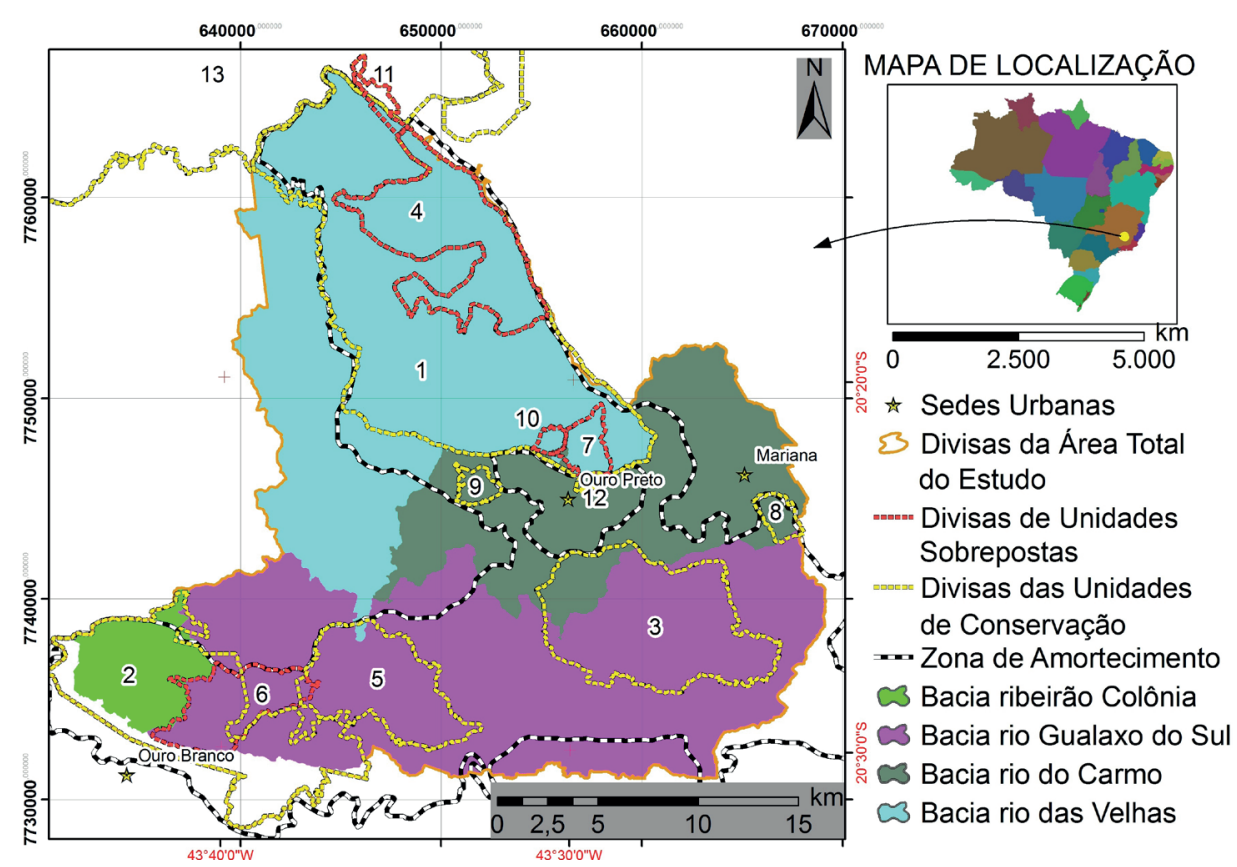

FIGURA 1 Área de estudo, delimitação das áreas protegidas e abrangência das bacias da região de Ouro Preto-MG, de acordo com a Tabela 1 (13. Área de Proteção Ambiental Sul da Região Metropolitana de Belo Horizonte - RMBH).

FIGURE 1 Study area, delimitation of protected areas and range of the basins of the Ouro Preto region, MG, according to Table 1 (13. Environmental Protection Area South of the Metropolitan Region of the Belo Horizonte - RMBH).

Para a análise da paisagem utilizaram-se imagens do sistema de satélites RapidEye AG, com $5 \mathrm{~m}$ de resolução espacial e cinco bandas espectrais; com data de passagem em junho de 2010. A escolha da imagem se deu pela necessidade de se obter precisão satisfatória em atendimento à escala de análise e, adicionalmente, pela frequência recorrente da passagem do satélite o que pode proporcionar futuras avaliações no local de estudo.

Com as imagens, fez-se um mosaico georreferenciado, utilizando o software Envi 4.7. Deste, retirou-se um subset onde se encontram as UCs, localizando-as nas bacias correspondentes aos afluentes do rio das Velhas, rio do Carmo, rio Gualaxo do Sul e ribeirão da Colônia, limitadas ao sul pela Serra de Ouro Branco e pequena parte do Parque Estadual da Serra de Ouro Branco e da RPPN Faz. Capanema.

A classificação do uso do solo, para fins de aplicação da metodologia das métricas, foi dividida em três classes: com floresta estacional semidecidual montana para os fragmentos maiores que 3 ha; o restante dos fragmentos de florestas foi agrupado aos campos naturais e campo com afloramento rochoso e água, compondo uma classe denominada de "vegetação natural 2"; e a última classe agrupou área urbana, eucalipto, pastagem, solo exposto, mineração, água e outros, e foi chamada de "uso antrópico".

O processo utilizado para a classificação do uso do solo foi "baseado em objetos" por meio do software ECognition Developer 8.0, do Laboratório de estudos e projetos em manejo florestal (LEMAF) da Universidade Federal de Lavras (UFLA). Para o processamento no software ECognition Developer 8.0 fez-se uma série de testes com inúmeros valores de entrada e, após essa avaliação, utilizaram-se os valores de: 250 para a escala de parâmetro; 0,6 para o fator de forma; e 0,3 para a compacidade. A escolha desse software se deu pela necessidade de se obter ganho de tempo na classificação das imagens e pela grande quantidade de ferramentas, 0 que proporciona maior agilidade no processo.

Completando o processo de classificação, procedeu-se com uma aferição de interpretação visual criteriosa, cuja edição contou com o auxílio de uma viagem de campo, realizada em junho de 20I I. Para a avaliação da qualidade do mapa de uso do solo, utilizouse a matriz de confusão e o índice de Kappa (k).

Em seguida, a imagem classificada foi exportada para vetor no formato SHAPE de polígonos para processamento no ArcGis 9.3. Para avaliar os fragmentos florestais na área de estudo, calcularam-se métricas da paisagem com o script V-Late I.I (LANG; TIEDE, 2003), que é uma extensão do ArcGis.

Para analisar $\circ$ formato dos fragmentos na paisagem, utilizou-se a média do índice de forma (MSI), média da razão perímetro área (RPA) e a média da 
dimensão fractal (MFRACT).

A seleção e a largura da borda utilizada para o cálculo das métricas foram baseadas nos estudos de Borges et al. (2004) e Pereira et al. (2007), cujos valores foram 50,75 e $100 \mathrm{~m}$.

Com a finalidade de avaliar estatisticamente a distribuição na paisagem dos fragmentos por classe de tamanho e visando a conhecer a distância do vizinho mais próximo (NNDist), procedeu-se com a divisão das manchas de vegetação nas seguintes amplitudes, em hectares: 3-5; 5-10; 10-20; 20-50;50-100; 100-200; 200-500; 500-I.000; I.000-5.000; 5.000-18.000. Foram calculados para o número de fragmentos (NP) e NNDist por classe: a média $(\mu)$, o coeficiente de variação $(C V)$ e o desvio padrão (DP).

Para o cálculo da métrica de proximidade (PROX) de MacGarigal et al. (2002), utilizaram-se os raios de 50, 75, 100 e $200 \mathrm{~m}$ para simulação.

\section{RESULTADOS E DISCUSSÃO}

As classes de cobertura do solo e suas respectivas áreas e porcentagens de ocupação na paisagem estão na Tabela 2 e o mapa está apresentado
TABELA 2 Área das classes de uso do solo na região de estudo em Ouro Preto, MG.

TABLE 2 Area of land use classes in the Ouro Preto region, MG.

\begin{tabular}{|c|c|c|c|}
\hline & \multirow{2}{*}{ Classes } & \multicolumn{2}{|c|}{ Área das Classes } \\
\hline & & (ha) & $\%$ \\
\hline \multirow[t]{2}{*}{1} & $\begin{array}{l}\text { Floresta Estacional Semidecidual } \\
\text { Montana (fragmentos }>3,0 \text { ha) }\end{array}$ & $42.287,30$ & 55,34 \\
\hline & Subtotal & $42.287,30$ & 55,34 \\
\hline \multirow{5}{*}{2} & Vegetação Natural 2 & & \\
\hline & Campo com Afloramento rochoso & $20.914,69$ & 27,37 \\
\hline & Campos Naturais & $3.043,28$ & 3,98 \\
\hline & $\begin{array}{l}\text { Vegetação (pequenos fragmentos } \\
\text { de florestas }<3,0 \text { ha) }\end{array}$ & 292,40 & 0,39 \\
\hline & Subtotal & $24.250,37$ & 31,74 \\
\hline \multirow{9}{*}{3} & Uso Antrópico & & \\
\hline & Pastagem & $6.187,51$ & 8,11 \\
\hline & Urbano & $1.170,33$ & 1,53 \\
\hline & Eucalipto & 896,27 & 1,17 \\
\hline & Outros & 844,12 & 1,11 \\
\hline & Solo Exposto & 415,98 & 0,54 \\
\hline & Água & 292,61 & 0,38 \\
\hline & Mineração & 64,21 & 0,08 \\
\hline & Subtotal & $9.871,03$ & 12,92 \\
\hline \multicolumn{2}{|c|}{ Total } & $76.408,70$ & 100,00 \\
\hline
\end{tabular}

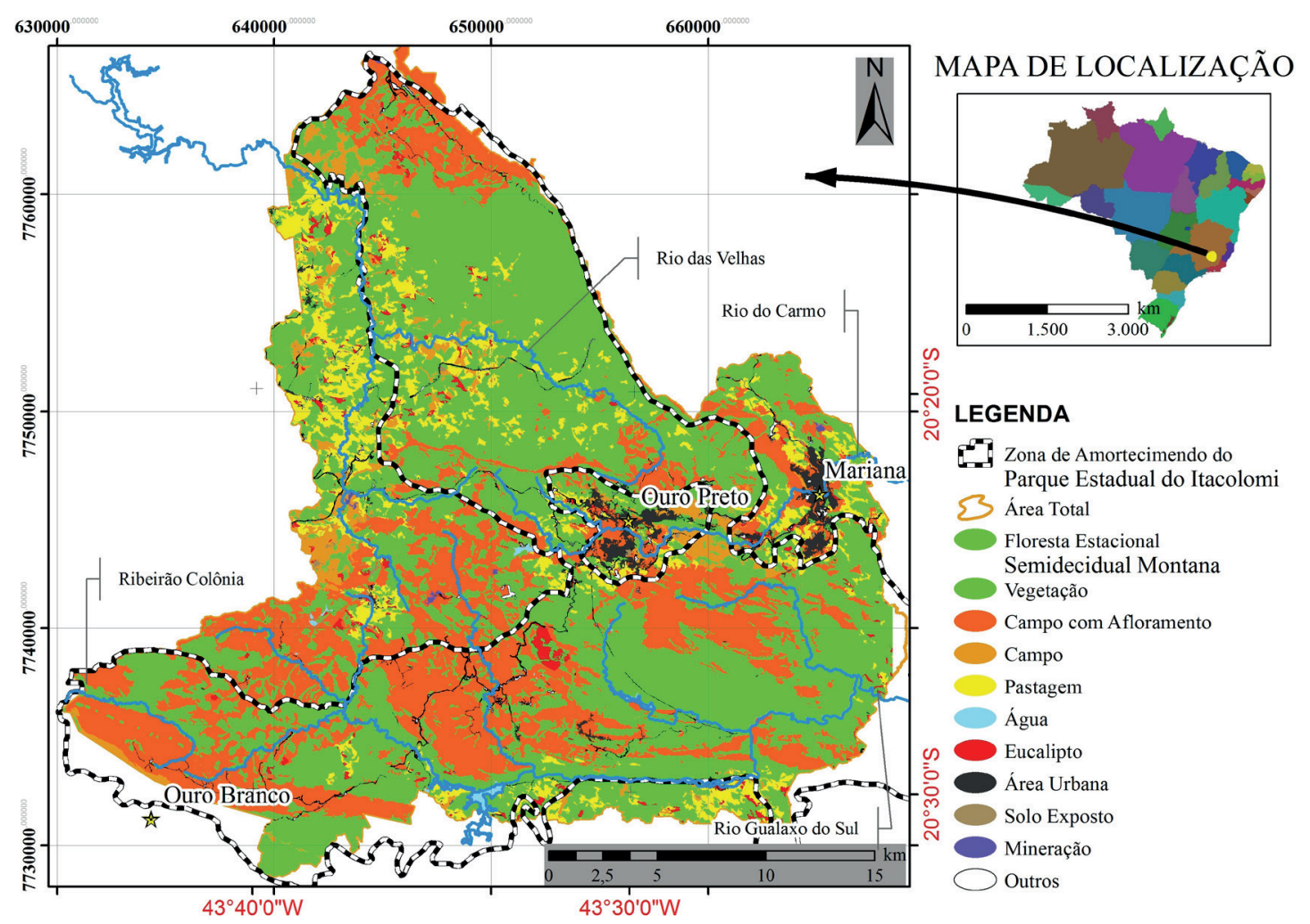

FIGURA 2 Mapa de classificação com usos do solo e os principais afluentes das bacias da região de Ouro Preto-MG.

FIGURE 2 Land use and the main watersheds tributaries classification map in the Ouro Preto region - MG. 
na Figura 2.

Pode-se observar que a floresta estacional semidecidual montana representa $55,34 \%$ da área, com 42.287,30 ha, seguida da classe vegetação natural 2 $(31,74 \%)$ e da classe uso antrópico (I2,9\%).

Em relação à qualidade global do mapa $\circ \mathrm{k}$ apresentou valor de 0,8840 que, segundo Landis e Koch (1977), corresponde a uma boa qualidade. Avaliando a matriz de confusão, observou-se que a classe Floresta Estacional Semidecidual Montana obteve percentuais acima do valor global, significando uma excelente classificação.

Segundo Hermuche e Felfili (20II), pode-se assumir que a defasagem entre a data do mosaico de imagens e $\circ$ trabalho de campo não interfere na delimitação dos fragmentos, pois estes estão situados sobre afloramentos rochosos e topografia acidentada, que funcionam como barreiras naturais ao avanço das atividades agropecuárias. $E$, também, a fiscalização nas áreas protegidas fortalece essa afirmação (SCALCO; GONTIJO, 2009).

As formações naturais da região somadas (classes I e 2) correspondem a $87,08 \%$ da área de estudo, mostrando que o conjunto de bacias ocupadas pelas UCs e áreas de preservação exercem suas funções de proteção dos ecossistemas locais, fornecendo habitats às espécies nativas e apresentando-se em longas extensões. Segundo Rezende et al. (2010), que avaliaram a cobertura da vegetação natural de 1985 a 2008 , com imagens Landsat e utilizando um subset que incluía, além da área deste estudo, todo o entorno da região, constatou-se que a área não sofreu alterações significavas ao longo do período.

Considerando as áreas naturais (classe I e 2), dentro da Zona de Amortecimento (ZA), encontraramse $62,63 \%$ da área total $(76.408,70 \mathrm{ha})$ e nas UCs correspondem a esse total $45,29 \%$. Pode-se observar que, no entorno da ZA, ocorrem $24,05 \%$ de áreas naturais. A fiscalização dentro das áreas com restrição de uso tem auxiliado a garantir a conservação ambiental, realidade também constatada por Scalco e Gontijo (2009) que trabalharam na APA Cachoeira das Andorinhas.

$\mathrm{Na}$ área de estudo, apenas o Parque Estadual do Itacolomi (PEI) tem a ZA regulamentada, possuindo restrições específicas às atividades antrópicas com a finalidade de minimizar impactos negativos sobre as mesmas (BRASIL, 2000). Como se pode visualizar na Figura I, esta ZA engloba a maioria das UCs, excluindo em sua área mais central a zona urbana de Ouro Preto e ao norte a RPPN.

O NP encontrado na área de estudo foi de 262.
Ao se calcular a MSI para os fragmentos (Tabela 3) da paisagem, obteve-se um valor de 3,035 que, comparados com os estudos de Pereira et al. (2007), que trabalharam com 20 fragmentos, dentre os quais a Serra de São José em Tiradentes, MG, onde obteve MSI igual a 3,57. Esse fragmento possui cerca de $\mathrm{I} .100 \mathrm{ha}$, muitas propriedades intercaladas às áreas de mata e tem uma forma alongada. Ainda, no estudo de Pereira et al. (2007), um fragmento (área de reserva legal da Universidade Federal de LavrasUFLA), cuja forma é próxima da circularidade, apresentou índice de forma igual a I,3I, valor muito próximo $(I, 2 I)$ do obtido para um dos fragmentos da área de estudos,

TABELA 3 Análise do formato dos fragmentos da região de Ouro Preto-MG.

TABLE 3 Shape analysis of the fragments in the Ouro Preto region - MG.

\begin{tabular}{lcccc}
\hline \multicolumn{1}{c}{ Classe } & NP & MSI & MPAR & MFRACT \\
\hline $\begin{array}{l}\text { Floresta Estacional } \\
\text { Semidecidual Montana }\end{array}$ & 262 & 3,035 & 0,029 & 1,382 \\
\hline
\end{tabular}

NP: número de fragmentos; MSI: média do índice de forma; MPAR: média do índice RPA; MFRACT: média da dimensão fractal.

cuja forma também é próxima da circular.

$\mathrm{Na}$ região de estudo, o valor encontrado para a MFRACT foi de I,382 que, comparado com os resultados de Gomide e Lingnau (2009), que trabalharam 14 áreas na Fazenda Experimental do Canguiri - PR onde obteve valor médio de $1,37 \mathrm{I}$, muito próximo ao do presente trabalho. A análise desse valor sugere fortes alterações na forma da paisagem, ou irregularidades naturais ou, ainda, alternância entre áreas da "classe floresta" com as de "vegetação natural" e "uso antrópico", estabelecendo na paisagem formas adversas para a preservação da biodiversidade ou complexas e irregulares, conforme os conceitos de fractais.

Analisando o efeito de borda, notou-se uma diminuição da área dos fragmentos e um aumento da fragmentação (Tabela 4), quando, na simulação, aumentou-se 0 efeito de borda de 50 para 75 e, finalmente, $100 \mathrm{~m}$. Simulando a borda em 50 metros tem-se uma redução no número de fragmentos de 262 para 122 (Áreas com fragmentos separados = DCA) e quando se utiliza o efeito de borda de 75 e $100 \mathrm{~m}$ o número de fragmentos reduz-se para 72 e $5 \mathrm{I}$, respectivamente. Consequentemente, as áreas internas dos fragmentos (TCA) diminuem cerca de $10 \%$ a cada 25 metros quando na simulação, aumenta-se a largura da borda. Na prática, o efeito de borda não é o mesmo em toda sua extensão, pois cada espécie (fauna e flora) possui habilidades intrínsecas que variam em função das 
TABELA 4 Simulação da largura de borda dos fragmentos para classe Floresta Estacional Semidecidual Montana da região de Ouro Preto-MG.

TABLE 4 Simulation of the fragments border width for the Seasonal semideciduous montane forest Class in the Ouro Preto region - MG.

\begin{tabular}{cccccc}
\hline $\begin{array}{l}\text { Largura de } \\
\text { borda }(\mathrm{m})\end{array}$ & DCA & NoCA & NCA & CA & TCA \\
\hline 50 & 122 & 18 & 1108 & & $28.674,63$ \\
75 & 72 & 91 & 827 & $42.287,30$ & $24.380,59$ \\
100 & 51 & 165 & 697 & & $20.944,25$ \\
\hline
\end{tabular}

DCA: fragmentos com áreas separadas do total de 262; NoCA: fragmentos sem área interna; NCA: número de áreas internas; $\mathrm{CA}$ : área do fragmento (ha); TCA: área interna do fragmento (ha)

especificidades das matrizes adjacentes, relevo etc.

O NP iniciais é de 262 , com a simulação do efeito de borda e somando as áreas formadas pela divisão dos fragmentos com a inserção do efeito de borda, esse valor cresceu na primeira simulação $(\mathrm{NP}=\mathrm{NCA}=1.108)$, isso ocorreu em função da subdivisão dos maiores fragmentos que possibilitaram a formação de apêndices no entorno do maior fragmento resultante, criando um grande número de fragmentos menores, estes, ainda, com a presença de áreas internas. Nas simulações seguintes, os fragmentos diminuem ou desaparecem com o aumento da borda simulada, respectivamente 827 e 697, para efeito de 75 e 100 metros. Esse comportamento dos dados é corroborado por Gomide e Lingnau (2009) e, segundo esses autores, as matas remanescentes são levadas à extinção e/ou à fragmentação, de acordo com o aumento do efeito borda, perdendo-se a conectividade estrutural.

Os efeitos da fragmentação e a criação de um grande número de áreas internas podem ser entendidos, a partir da complexidade da estrutura geométrica dos fragmentos na paisagem, que se comportam como fractais (BEVILACQUA et al., 2008), as áreas que se separaram com a simulação da borda estão representadas por apêndices, os quais Houaiss (2009) define como parte pertencente a outra maior e que a completa. Esses apêndices numericamente crescem ou desaparecem, a partir da fragmentação e seu respectivo efeito de borda.

$O$ efeito de borda atua sobre os fragmentos e suas irregularidades, transformando figuras geométricas complexas em superfícies que tendem à circularidade e, assim, ao longo das simulações, obtêm-se valores menores para o NCA.

Como dito anteriormente, com a simulação (aumento) de largura de borda há diminuição do número de fragmentos com áreas separadas (DCA) dos fragmentos maiores. No sentido inverso, há aumento do número de fragmentos sem áreas internas (NoCA), concordando com as tendências encontradas por Gomide e Lingnau (2009) e Pereira et al. (2007).

Ao analisar os fragmentos por classes de tamanho (Tabela 5), observou-se uma grande concentração $(85,49 \%)$ em número de fragmentos (NP) menores que 50 ha, correspondendo a $6 \%$ do total da área. Entretanto, a classe de 5.000 a 18.000 ha abrange, aproximadamente, $69 \%$ da área total e possui quatro fragmentos. Pütz et al. (20l I) sugeriram que a fragmentação altera a estrutura da floresta, principalmente para os fragmentos florestais menores do que 25 ha, onde os fragmentos florestais são levados para os estados iniciais de sucessão. Ribeiro et al. (2009) encontraram o percentual de $85 \%$ na Mata Atlântica do

TABELA 5 Número de fragmentos por classe de área e distância do vizinho mais próximo da região de Ouro Preto-MG.

TABLE 5 Number of fragments per area class and distance from the nearest neighbor in the Ouro Preto region - MG.

\begin{tabular}{|c|c|c|c|c|c|c|c|}
\hline $\begin{array}{l}\text { Classes de área dos } \\
\text { fragmentos (ha) }\end{array}$ & NP & $\mu$ área (ha) & DP área (ha) & CV\% & $\mu$ NNDist $(\mathrm{m})$ & DP NNDist (m) & CV\% \\
\hline 3 a 5 & 66 & 3,87 & 0,57 & 14,69 & 117,93 & 146,51 & 124,23 \\
\hline 5 a 10 & 78 & 7,19 & 1,54 & 21,46 & 84,32 & 106,45 & 126,24 \\
\hline 10 a 20 & 49 & 14,31 & 2,57 & 17,92 & 58,32 & 88,15 & 151,16 \\
\hline 20 a 50 & 31 & 32,86 & 8,14 & 24,78 & 33,15 & 32,01 & 96,56 \\
\hline 50 a 100 & 13 & 69,09 & 14,70 & 21,28 & 20,20 & 24,64 & 121,97 \\
\hline 100 a 200 & 15 & 136,56 & 23,12 & 16,93 & 17,67 & 21,64 & 122,46 \\
\hline 200 a 500 & 2 & 271,44 & 45,43 & 16,74 & 44,39 & 19,33 & 43,54 \\
\hline 500 a 1.000 & 4 & 715,49 & 121,95 & 17,04 & 5,09 & 3,29 & 64,62 \\
\hline 1.000 a 5.000 & 2 & $2.244,87$ & $1.170,37$ & 52,14 & 10,30 & 7,78 & 75,58 \\
\hline 5.000 a 18.000 & 2 & $14.455,12$ & $4.918,49$ & 34,03 & 3,78 & 0,87 & 22,99 \\
\hline Total Geral & 262 & 161,40 & $1.312,13$ & 812,96 & 72,18 & 107,36 & 148,74 \\
\hline
\end{tabular}

NP: número de fragmentos; $\mu$ : média; DP: desvio padrão; CV\%: coeficiente de Variação; NNDist: distância do vizinho mais próximo. 
Brasil, para esses fragmentos menores que 25 ha.

Em relação à localização dos fragmentos na paisagem, as classes de menor tamanho ( $<50$ ha) encontram-se espalhadas na paisagem, com 42,81\% de suas respectivas áreas dentro da ZA e destas 33,68\% dentro das UCs. Nas classes intermediárias (100 a 500 ha) a distribuição segue padrão parecido, com $43,64 \%$ dentro da ZA, porém possuem apenas 5,64\% correspondentes as UCs. Essas classes menores situamse, principalmente, fora das áreas protegidas e são constituídas de reservas legais ( $R L$ ) das propriedades privadas, pequenas manchas de matas ciliares e vegetação em APP nas encostas preservadas das propriedades.

Ao contrário das classes de menor amplitude, as classes de 1.000 a 18.000 ha contêm grande parte de seus fragmentos dentro das áreas protegidas, com $83,95 \%$ da área dentro da ZA e, destes, $62,38 \%$ nas UCs, com destaque para a APA Andorinhas e Parque Estadual do Itacolomi, que apresentam as maiores áreas, fato que corrobora com a função das UCs na região do estudo.

Além da preservação de grandes áreas, fazse necessário avaliar outros fatores como a qualidade dos fragmentos. Segundo Rambaldi e Oliveira (2003), a preservação possui interdependência com estrutura, riqueza de habitats, grau de isolamento, natureza do entorno, relações interespecíficas (predador-presa) e intraespecíficas, incluindo o comportamento social de animais e interferências antrópicas. Essa interdependência justifica futuros estudos mais específicos, com a finalidade de melhor avaliar o estado de conservação das áreas naturais presentes na área de estudo.

$\mathrm{Na}$ avaliação estatística dos fragmentos (Tabela 5), observou-se que o desvio padrão da média (DP) e o coeficiente de variação $(\mathrm{CV})$, foram menores nos fragmentos com tamanho inferior a 1.000 ha $(0,57$ a 121,95, respectivamente para a classe de 3 e 1.000 ha), onde houveram menores variações nas dimensões dos fragmentos; entretanto, a variação nas duas últimas classes foi maior (I.170,37 e 4.918,49 ha) do que para as classes anteriores, isso ocorreu, em decorrência da presença de um fragmento de tamanho muito superior (correspondendo a uma área de I7.930 ha), na região da APA Cachoeira das Andorinhas e entorno.

Ao se considerar a média de NNDist, todos os fragmentos acima de 50 ha estão mais próximos de algum fragmento, mesmo que de tamanho variado, com distância média inferior a $45 \mathrm{~m}$. O DP e CV de NNDist se mostraram muito elevados, com apenas a classe de 5.000 a 18.000 há, obtendo baixo valor $(0,87 \mathrm{~m})$ de DP e, aproximadamente, $23 \%$ de CV (Tabela 5). Isso ocorreu, pelo fato de os maiores fragmentos ocuparem grande parcela da paisagem, às vezes divididos apenas por estradas ou rios (os quais, às vezes, são totalmente cobertos pelas copas das árvores), mantendo-se sempre próximos a algum fragmento.

O índice proximidade (PROX) e NNDist, para Cushman et al. (2008), são componentes universais para análise da paisagem, utilizados no grau de isolamento dos fragmentos e conectividade da paisagem. Ao se comparar à média do índice PROX para todos os fragmentos (Tabela 6), procedendo com simulação para os quatro raios diferentes, percebeu-se um aumento progressivo nos valores obtidos.

TABELA 6 Simulação do índice de proximidade (PROX) para as distâncias pré-definidas da região de Ouro Preto-MG.

TABLE 6 Simulation of proximity index (PROX) for pre-defined distances in the Ouro Preto region - MG.

\begin{tabular}{ccc}
\hline $\begin{array}{c}\text { Distância pré-definida } \\
(\mathrm{m})\end{array}$ & NP & Média PROX \\
\hline 50 & & 40,12 \\
75 & 262 & 40,34 \\
100 & & 40,39 \\
200 & & 40,47 \\
\hline
\end{tabular}

NP: número de fragmentos; PROX: índice de proximidade.

A média de NNDist para todos os fragmentos é 72 , I 8 m, com o máximo de 603,67 m e o mínimo de 0,84 $\mathrm{m}$, que geraram um alto DP (Tabela 5). Dentre todos os fragmentos estudados, 73 apresentam NNDist acima da média (72, $18 \mathrm{~m})$. Desse modo, as simulações, utilizando raios maiores que a média NNDist para a inclusão de fragmentos ao índice PROX, incorporam os fragmentos mais isolados, que são desprezados quando o raio de busca é menor. Esses fragmentos mais isolados possuem função de stepping stones. Em relação a extensões utilizadas para as avaliações experimentais, nos estudos de Develey e Metzger (2006) são considerados razoáveis para o grupo das aves de sub-bosque, que são limitadas para grandes distâncias.

Visando à conservação, segundo Creed (2006), os planos de manejo florestais devem considerar que perturbações são importantes para estruturar as comunidades biológicas, sendo responsáveis pela manutenção da biodiversidade em vários ecossistemas. Porém, as perturbações com origem antrópica intensificam a fragmentação natural da paisagem, e têm consequências ambientais severas, que vão, desde os impactos no solo até o dossel da floresta. Segundo Olifiers e Cerqueira (2006) as espécies são afetadas distintamente, os habitats ditos negativos para algumas espécies passam a ser de boa qualidade para outras. A 
perda de cobertura vegetal fica evidente ao se comparar a área externa e interna da ZA.

Nesse sentido, o planejamento da paisagem deve considerar aqueles que residem e têm interesse direto na área. Os projetos de meio ambiente e a população devem coexistir, respeitando os conceitos do desenvolvimento sustentável.

\section{CONCLUSÕES}

A área do estudo apresenta-se em bom estado de conservação, considerando a cobertura vegetal natural e estrutural, especialmente nas Unidades de Conservação. No interior da área de estudo, encontramse grandes fragmentos e outros de tamanhos menores ao longo de toda a bacia, mesmo em locais submetidos à forte pressão antrópica.

A aplicação dos índices da paisagem mostrou que, entre os grandes fragmentos, observam-se muitas médias e pequenas áreas com cobertura vegetal arbórea, funcionando como stepping stones por todas as bacias da área de estudo; esses fragmentos menores têm grande importância na conectividade entre os fragmentos e as áreas de campos naturais e plantados, espalhados na paisagem.

No primeiro momento, as áreas ocupadas por bordas ampliam a fragmentação; por outro lado, como resultado da redução nas dimensões individuais decorrentes do efeito de borda, observa-se a suavização das formas complexas que pode conduzir à extinção de parte dos fragmentos.

A existência de Zona de Amortecimento no parque, apresentou-se importante para a preservação de áreas de vegetação natural no seu entorno, embora pontos vulneráveis sejam encontrados próximos às áreas de pastoreio e mineração, com destaque para as áreas de campo no município de Ouro Branco e áreas de pastoreio localizadas no interior da APA.

Fortes pressões antrópicas estão presentes fora da Zona de Amortecimento, observadas pela presença de grande quantidade de áreas com solo exposto; em alguns casos com degradação em formas de voçorocas, mostrando a importância da Zona de Amortecimento para a preservação e conservação das áreas naturais.

\section{AGRADECIMENTOS}

Ao Instituto Estadual de Florestas (IEF), Regional Centro Sul (município de Ouro Preto) e à Coordenação de Aperfeiçoamento de Pessoal de Nível Superior
(CAPES).

\section{REFERÊNCIAS}

BEVILACQUA, L.; BARROS, M. M.; GALEÃO, A. C. N. R. Geometry, dynamics and fractals. Journal of the Brazilian Society of Mechanical Sciences and Engineering, Rio de Janeiro, v. 30, p. II-2I, 2008.

BORGES, L. F. R.; SCOLFORO, J. R.; OLIVEIRA, A. D. Inventário de fragmentos florestais nativos e propostas para seu manejo e o da paisagem. Cerne, Lavras, v. 10, n. I, p. 22-38, 2004.

BRASIL. Lei n 9.985, de 18 de julho de 2000. Institui o Sistema Nacional de Unidades de Conservação da Natureza e dá outras providências. Diário Oficial [da] República Federativa do Brasil, Brasília, I0 jul. 2000. Seção I, p. I.

CARVALHO, L. M. T. de. Detecção de modificações na cobertura do solo. In: CARVALHO, L. M. T. de; SCOLFORO, J. R. S. (Ed.). Inventário florestal de Minas Gerais: monitoramento da flora nativa 2005-2007. Lavras: UFLA, 2008. p. I-24.

CREED, J. C. Perturbações em comunidades biológicas. In: ROCHA, C. F. D.; BERGALHO, H. G. van; SLUYS, M.; ALVES, M. A. S. (Ed.). Biologia da conservação: essências. São Carlos: Rima, 2006. p. 183-210.

CUSHMAN, S. A.; MCGARIGAL, K.; NEEL, M. C. Parsimony in landscape metrics: strength, universality, and consistency. Ecological Indicators, Amsterdam, v. 8, p. 69I-703, 2008.

DEVELEY, P. F; METZGER, J. P. Emerging threats to birds in Brazilian Atlantic forests: the roles of forest loss and configuration in a severely fragmented ecosystem. In: LAURANCE, W. F.; PERES, C. A. (Ed.). Emerging threats to tropical forests. Chicago: University of Chicago, 2006. p. 269-290.

FUNDAÇÃO JOÃO PINHEIRO. Plano de conservação, valorização e desenvolvimento de Ouro Preto e Mariana. Belo Horizonte, 1975.

GOMIDE, L. R.; LINGNAU, C. Simulação espacial de uma paisagem sob o efeito borda. Floresta, Curitiba, v. 39, n. 2, p. 44 I-455, abr./jun. 2009.

HERMUCHE, P. M.; FELFILI, J. M. Relação entre NDVI e florística em fragmentos de floresta estacional decidual no Vale do Paranã, Goiás. Ciência Florestal, Santa Maria, v. 2I, n. I, p. 4I-52, jan./mar. 20 II.

HOUAISS, L. Houaiss eletrônico. Rio de Janeiro: Objetiva, 2009.

INSTITUTO DE PESQUISAS ESPACIAIS; FUNDAÇÃO SOS MATA ATLÂNTICA. Atlas dos remanescentes florestais da Mata Atlântica, período 2008-20I0. São Paulo, 20I I. Disponível em: <http://www.sosmatatlantica. 
org.br/>. Acesso em: 3I jan. 2012.

LANDIS, J. R.; KOCH, G. G. The measurement of observer agreement for categorial data. Biometrics, Arlington, v. 33, p. 159-174, 1977.

LANG, S.; TIEDE, D. vLATE extension für ArcGIS: vektorbasiertes tool zur quantitativen. Landschaftsstrukturanalyse: ESRI, 2003.

MCGARIGAL, K.; CUSHMAN, S. A.; NEEL, M. C.; ENE, E. Fragstats: spatial pattern analysis program for categorical maps. Version 3.3 build 5. Amherst: University of Massachusetts, 2002.

METZGER, J. P. W. O que é ecologia de paisagens? Biota Neotropica, Campinas, v. I, n. I/2, p. I-9, 200 I.

MOULTON, T. P.; SOUZA, M. L. de. Conservação com base em bacias hidrográficas. In: ROCHA, C. F. D.; BERGALHO, H. G. van; SLUYS, M.; ALVES, M. A. S. (Ed.). Biologia da conservação: essências. São Carlos: Rima, 2006. p. 157-182.

OLIFIERS, N.; CERQUEIRA, R. Fragmentação de habitat: efeitos históricos e ecológicos. In: ROCHA, C. F. D.; BERGALHO, H. G. van; SLUYS, M.; ALVES, M. A. S. (Ed.). Biologia da conservação: essências. São Carlos: Rima, 2006. p. 26I-280.

OLIVEIRA FILHO, A. T. de; SCOLFORO, J. R. S.; OLIVEIRA, A. D. de; CARVALHO, L. M. T. de. Definição e delimitação de domínios e subdomínios das paisagens naturais do Estado de Minas Gerais. In: SCOLFORO, J. R.; CARVALHO, L. M. T. de (Org.). Mapeamento e inventário da flora nativa e dos reflorestamentos de Minas Gerais. Lavras: UFLA, 2006. v. I, p. 2 I-35.

PEREIRA, J. A. A.; OLIVEIRA-FILHO, A. T.; LEMOS-FILHO, J. P. Environmental heterogeneity and disturbance by humans control much of the tree species diversity of Atlantic montane forest fragments in SE Brazil. Biodiversity and Conservation, Dordrecht, v. 16, p. |76|-|784, 2007.
PÜTZ, S.; GROENEVELD, J.; ALVES, L. F; METZGER, J. P.; HUTH, A. Fragmentation drives tropical forest fragments to early successional states: a modelling study for Brazilian Atlantic forests. Ecological Modelling, Amsterdam, v. 222, p. 1986-1997, 2011.

RAMBALDI, D. M.; OlIVEIRA, D. A. S. de (Org.). Fragmentação de ecossistemas: causa, efeitos sobre a biodiversidade e recomendações de políticas públicas. Brasília: MMA/SBF, 2003. 510 p.

REZENDE, R. A.; PRADO-FILHO, J. F.; SOBREIRA, F. G.; SANTOS, T. F. Dinâmica da cobertura do solo no extremo sul da Serra do Espinhaço: métricas da paisagem e a conectividade entre áreas protegidas. Geografia, Rio Claro, v. 35, n. 3, p. 683-699, 2010.

RIBEIRO, M. C.; METZGER, J. P.; MARTENSEN, A. C.; PONZONI, F. J.; HIROTA, M. M. The Brazilian Atlantic Forest: how much is left, and how is the remaining forest distributed?: implications for conservation. Biological Conservation, Oxon, v. I42, p. I|4|-II53, 2009.

SCALCO, R. F; GONTIJO, B. M. Mosaico de unidades de conservação: da teoria à prática: o caso do mosaico de unidades de conservação da APA Cachoeira das Andorinhas, Ouro Preto/MG. Geografias, Belo Horizonte, v. 5, n. 2, p. 75-92, jul./dez. 2009.

TABARELLI, M.; GASCON, C. Lições da pesquisa sobre fragmentação aperfeiçoando políticas e diretrizes de manejo para a conservação da biodiversidade. Megadiversidade, Belo Horizonte, v. I, n. I, p. |8I-|88, jul. 2005.

UNIVERSIDADE FEDERALDEVIÇOSA;FUNDAÇÃO CENTRO TECNOLÓGICO DE MINAS GERAIS; UNIVERSIDADE FEDERAL DE LAVRAS; FUNDAÇÃO ESTADUAL DO MEIO AMBIENTE. Mapa de solos do Estado de Minas Gerais Belo Horizonte. FEAM, MG, p. 49, 20I0. Disponível em: $<$ http://www.feam.br/noticias/ //949-mapas-de-solo-doestado-de-minas-gerais >. Acesso em: 02 mar. 2010.

VELOSO, H. P.; RANGEL-FILHO, A. L. R.; LIMA, J. C. A. Classificação da vegetação brasileira, adaptada a um sistema universal. Rio de Janeiro: Fundação IBGE, I99I. 123 p. 
